\title{
Dengue Fever: A Review Article
}

\author{
R. Prem Kumar ${ }^{1}$, R. Sunith ${ }^{2 *}$, A. Karthikeyan ${ }^{3}$ and V. Pradeep Kumar ${ }^{4}$ \\ ${ }^{1}$ Veterinary Dispensary, Madanahalli, Kolar, Karnataka, India \\ ${ }^{2}$ Department of Veterinary Public Health and Epidemiology, \\ Veterinary College, Gadag, India \\ ${ }^{3}$ Department of Veterinary Public Health and Epidemiology, IVRI, Bareilly, India \\ ${ }^{4}$ Veterinary Dispensary, Alabanur, Raichur, Karnataka, India \\ *Corresponding author
}

Keywords

Dengue Fever, Aedes, Rainfall, Clinical symptoms, Treatment

Article Info

Accepted:

15 December 2019

Available Online:

20 January 2020

\section{A B S T R A C T}

Dengue fever is the one of the rapidly expanding mosquito-borne viral disease in the world, with high mortality and morbidity rates especially in tropical and subtropical regions. The mosquito involved in the transmission of dengue is Aedes. The circulation of dengue disease is influenced by various factors such as, topography, rainfall, temperature and rapid urbanization or globalization. The clinical symptoms range from unapparent to severe forms and fatal outcomes. Dengue is a most important public health problem due its quick expansion globally and its burdens are currently unfulfilled because of absence of precise treatment, easy diagnostic method for the early phase of infection and successful and wellorganized vector control system.

\section{Introduction}

Dengue is a disease of public health importance and it is a mosquito-borne flavivirus infection caused by four related but antigenically distinct dengue viruses (DENVs, serotypes 1-4). Dengue is known as a "Disease of Poverty" as it is most closely associated with poor populations in tropical and subtropical regions (Rigau-Perez, 1998;
WHO, 2012). The World Health Organization classifies dengue fever as one of the world's 17 neglected tropical diseases (NTDs).

Dengue is endemic in more than 100 countries (mostly in Southeast Asia and South America) and it is rapidly disseminating to new regions with epidemics of increasing magnitude and severity (WHO, 2015). Approximately 390 million people worldwide 
are infected per year; only 96 million exhibit severe symptoms and approximately 20,000 die (Beltran-Silva, 2016). Dengue Fever (DF) and Dengue Hemorrhagic Fever (DHF) have been reemerging and expanding globally over the past 50 years at alarming rates (WHO, 2011; Thai and Anders, 2011). The dengue infected persons either have no symptoms or have mild self-limited disease (including fever, headache, retroocular pain, muscle and joint pain, nausea, vomiting, and rash); a small proportion of infections progress to severe illness, with rapid onset of capillary leakage accompanied by bleeding, thrombocytopenia, and liver injury (RigauPérez, 1998; Paixao et al., 2016). Presently, treatment being purely supportive as there is no therapeutic option. In most cases disease is managed by fluid replacement. In addition to early and accurate diagnosis, early markers of progression to severe disease are urgently needed. (Rosenberger et al., 2016; Muller et al., 2017). Most of the infected persons are either are asymptomatic or have a mild undifferentiated fever hence diagnosis on the basis of clinical symptoms is not reliable and hence laboratory diagnosis has to be carried out (Endy et al., 2002). This review highlights the etiology, transmission and risk factors associated with the transmission, methods for early diagnosis, treatment and prevention and control measures.

\section{Etiology}

Dengue virus (Flaviviridae family, Flavivirus genus) has four different subtypes (DENV-1, DENV-2, DENV-3 and DENV-4) and it is transmitted by infected mosquitoes Aedes spp. (Aedes aegypti and albopictus) (Simmons et al., 2012). Infection with one serotype provides long-lasting immunity against that particular serotype, whereas cross-immunity to the other serotypes is temporary (Rigau-Perez, 1998; Paixao et al., 2016).
Characteristic features of dengue genome are as follows: a spherical particle of small size (diameter 40-50 nm) covered by glycoprotein envelope and single-strand messenger (positive) sense RNA of approximately $11 \mathrm{~kb}$ in length (Henchal and Putnak, 1990). The envelope functions in hemagglutination phenomenon, neutralization, and interaction between virus and host cell during the early phase of infection. The Dengue virus and its soluble membrane-associated NS1 activate human complement system and express in blood during the early phase of infection. Viral load, terminal SC5b-9 complement complex and NS1 antigenemia have been shown to be associated with dengue severity (Roth et al., 2014).

\section{Transmission}

Aedes aegypti, a cosmotropical mosquito, acts as the principle vector for dengue virus (DENV) which is found in urban areas of many tropical and semitropical regions of the world. It is extremely anthropophilic and feeds on blood and it breeds chiefly in stagnant water which is found in discarded containers and car tires. Female mosquitoes play an important role in transmition of virus to humans (WHO, 2011; WHO, 2012). The life cycle of mosquito is having four discrete stages that is egg, larva, pupa and adult. The duration of the larval and pupal stage is 7-9 and 2-3 days respectively at at $25^{\circ} \mathrm{C}$. After feeding on an infected host, the transmission of virus can occur after an 8-10day extrinsic incubation period (EIP) (WHO, 2011; Thai and Anders, 2011). The speed of larval growth increases with rise in temperature and the succeeding emergence of adult vectors as well as the vector-biting rate. The rise in temperature by $2^{\circ} \mathrm{C}$ will reduce the EIP of DENV, which intern increases the frequency of mosquito bite (Focks and Barrera, 2007). Another mosquito vector Aedes albopictus, which was initially restricted to Asia, 
extended its global range now a day's and contributed to the spread of the dengue (Kuehn, 2014 and Rezza, 2014).

\section{Risk factors associated with dengue}

Host immunity, vector capacity, circulating DENV, weather or climate, dengue control capacity and population movement is the risk factors involved in the transmission of Denue. The climatic factors influence the dengue epidemiology because of its indirect impact on lifecycle of mosquitoes and on incubation periods of DENV within mosquitoes (Morin et al., 2013). Stagnated water in open can become a breeding ground for mosquitoes which carry dengue virus (Messina et al., 2014). The urban expansion and rapid population growth has lead to resurgence of dengue. Unplanned urban expansion has led to the severe constraints on community facilities, predominantly water supply and solid waste disposal, thus increasing the breeding potential of the vector species which intern causes the exposure of large number of human population to the mosquito vector Ae. aegypti (WHO, 2009; Thai and Anders, 2011). The lack of mosquito control infrastructure, increased air travel and globalization of trade has been extensively contributed to the introduction of all dengue virus serotypes to most population centers of the world (Horstick, 2010).

The globalization and travel has led to hyper endemic transmission of all four dengue serotypes throughout the tropics (Morin, 2015). The temperature and rainfall affect the spread of mosquito vectors and virus transmission (Shuman, 2010). It is forecasted that by the year 2100, the global warming leads to an increase in global temperatures by 2 and $4.5^{\circ} \mathrm{C}$ and could have a perceptible impact on vector-borne diseases. Climate change is predicted to lead to a considerable increase in populations at risk of dengue and could expose an additional two billion people to dengue transmission by 2080 (McMichael et al., 20006). Studies also anticipate that global climate change can have an impact on sylvatic dengue virus affecting wild animals, as compared to urban DENV transmission and lead to cross-species transmission of DENV into humans (Vasilakis et al., 2011).

\section{Clinical Manifestations}

\section{Undifferentiated fever}

This phase is mostly commonly seen in the primary infection and also after the initial secondary infection. Clinically, it is tedious to distinguish from other viral diseases and remains undiagnosed.

\section{Dengue fever}

Dengue Fever often observed after primary and secondary infections. It is most commonly noticed in adults and older children. It is characterized by a biphasic, high-grade fever remains for a period 3 days to 1 week (Narayanan et al., 2002). The other symptoms include severe headache (mainly retrobulbar), loss of appetite, vomition, abdominal pain and diarrhea. Due to the associated myalgia and joint pain it is also known as breakbone fever (Whitehorn and Farrar, 2010 and Narayanan et al., 2002). Nearly $50-82 \%$ of infected individuals encounter the lesions of cutaneous rash due to capillary dilatation, maculopapular or morbilliform eruption (Itoda et al., 2006; Radakovic-Fijan et al., 2002). Bleeding is rarely seen in DF, even though epistaxis, gingival bleeding, extensive menstruation, petechiae/purpura and gastrointestinal tract hemorrhage can occur (Ahmed et al., 2001).

\section{Dengue Shock Syndrome (DSS)}

DSS is defined as DHF accompanied by an unstable pulse, narrow pulse pressure $(<20$ $\mathrm{mmHg}$ ), restlessness, cold, clammy skin and 
circumoral cyanosis. High mortality rate is seen in patients with DSS due to multi organ damage and disseminated intravascular coagulation. The shock remains only for a short period of time and these patients rapidly recovers from the disease with sympathetic treatment (Gurugama et al., 2010; Shivpuri and Shivpuri, 2011).

\section{Laboratory diagnosis of dengue virus infection}

Laboratory diagnosis of dengue virus infection can be made by the detection of specific virus, viral antigen, genomic sequence and/or antibodies (Guzman and Kouri, 2003).

Virus isolation has been considered as the conventional diagnostic test for detecting the DENV infection. For virus isolation, samples taken from patients are cultured in various cell lines of either mammalian (BHK-21, Vero and LLCMK2 cells) or mosquito (CLA1, AP-61, Tra-284, C6/36, AP64 cells) origin or in live mosquitoes (Shu et al., 2004, Colombo, 2016). However, the rapid diagnostic tests such as reverse-transcription polymerase chain reaction (RT-PCR) and NS1 antigen-capture enzyme-linked immunosorbent assays (ELISAs) have replaced the traditional diagnostic method (Shu et al., 2004). Molecular techniques such as RT-PCR and nucleic acid hybridization have been applied successfully for detecting the DENV infection. A highly sensitive 2-step step heminested RT-PCR test was initially reported by Lanciotti et al., (1992) and it was then modified to a single-step multiplex realtime RT-PCR assay, which was used globally. The PCR based methods sensitive, specific, less time consuming, less complicated and cheaper than virus isolation methods (Deubel et al., 1990). Detection of NS1 in patient blood employing an antigen-capture ELISA technique was first described in 2000 (Alcon et al., 2002). The NS1 viral protein which is secreted from infected cells and found in higher concentration in the blood of infected person can be used as an ideal diagnostic target. It can be detected from onset of disease to 9days or longer after the occurrence of disease. This protein can be diagnosed along with viral RNA and before an antibody response is initiated in primary infections (Young et al., 2000; Alcon et al., 2002). The NS1 as a diagnostic tool has high sensitivity and specificity (Simmons et al., 2007).

The dengue infecting serotype is generally detected by quantifying a four-fold or greater rise in antibodies to that particular serotype. The serological tests are comparatively simple and some reagents are commercially available (WHO, 1997; Teles, 2005). Hem agglutination Inhibition test (HI) is simple and more sensitive than electrophoretic methods, being able to detect less than 1 $\mathrm{mg} / \mathrm{ml}$ of antibodies (Roitt et al., 2001). This test is suitable for epidemiological studies as some HI antibodies may persist 50 years or more (WHO, 2011). A major disadvantage of the method is the requirement for chemical pretreatment to remove nonspecific inhibitors of haemagglutination and further absorption with red blood cells to remove non-specific agglutinins. Moreover, the test usually does not discriminate infections caused by closely related flaviviruses, nor is it serotype-specific, due to cross-reacting dengue antibodies. In case of Complement Fixation Test (CFT) unlike the IgM or the HI antibodies, which appear within 5-7 days, the CF antibody appears 7-14 days after the onset and persists for shorter period (WHO, 1999). CFT is the least sensitive serological test for dengue. The Enzyme Immuno Assay (EIA) has been employed to detect and distinguish $\mathrm{IgG}$ and IgM antibodies to dengue and it is highly sensitive. This technique is extensively used since it is available readily as a commercial kit, allowing screening of large number of 
samples in a short period of time (Roitt et al., 2001). The main advantage of the test is its ability to differentiate primary from secondary infections based on the IgM/IgG ratio. The World Health Organization recommends classical dengue monoclonal antibody (IgM)-capture EIA (MAC-EIA), for serological diagnosis of dengue infections as it is inexpensive, simpler and faster and provides more information than other serological tests (Innis et al., 1989; Muller et al., 2017).

\section{Dengue treatment}

Dengue infection is associated with complex clinical symptoms thus; therapeutic management should be simple, low cost effective in saving lives through correctly performed and timely institutionalized interventions (WHO, 2009). There are no effective antiviral drugs for dengue hence clinical management focuses on supportive treatment, with particular importance on cautious fluid management (Simmons et al., 2012; WHO, 2009). Individuals with early febrile phase with no complications may recover from disease without the need of hospital admission with intensive daily monitoring for any bleeding. Hospitalization is required for the patients with early stage of plasma leakage or critical phase (Simmons et al., 2012; WHO, 2009).

During critical stage, Sensible and optimal fluid resuscitation is crucial to sustain organ perfusion (Whitehorn et al., 2014). In case of DSS, isotonic crystalloid solutions, such as $0.9 \%$ normal saline, Ringer's lactate or Hartmann's solution can be employed for initial according to WHO guidelines on fluid management algorithm (Simmons et al., 2012; WHO, 2009; Wills et al., 2005). The main aim of fluid resuscitation is to avoid complications of vascular leakage and hypovolemic shock (Chan and Ooi, 2015). In spite of fluid therapy intervention, it was anticipated that $30 \%$ of DSS cases experienced recurrent episodes of shock (Whitehorn et al., 2014).Transfusion of blood product such as, packed red cell, platelets or fresh-frozen plasma, can be lifesaving for patients with severe bleeding (Simmons et al., 2012; Chan and Ooi, 2015).

In phyto medicine, several sulfated polysaccharides extracted from seaweeds have been studied and they have high antiviral action against dengue virus (Damonte et al., 2004). In modern medicine, glycyrrhizin, ribavirin and 6-azauridine are found to have cytostatic and inhibitory effects on the dengue virus (Crance et al., 2003). Currently clinical research is going on to find the specific antidengue drugs (Wilder-Smith, 2010; Wiwanitkit, 2017).

\section{Prevention and Control}

Primarily the dengue infection can be controlled by the control of dengue vectors which can be aimed against the immature aquatic stages (larvae and pupae) or the adult mosquitoes. Direct vector control measures include, use of insecticides to kill the mosquitoes or prevent them from biting by employing repellents. Environmental modification or sanitation improvements that reduce potential larval development sites or house improvements that prevent mosquito entry can be used as indirect vector control methods (WHO, 2009 and Kuehn, 2014). For space-spraying and larviciding require trained personnel in contrast, the reduction in potential larval development sites can be achieved with householders. Some of the community based efforts like empowering of affected and other communities through education and advocacy can mobilize and mount effective control operations (WHO, 2009).

In conclusion, dengue is a major public health problem in almost all countries located in 
tropical and subtropical regions. The social and economic burden of mosquito-borne dengue infection is extensively alarming. The morbidity and mortality of dengue and other mosquito-borne viral infection can be reduced by performing early identification of high-risk patients and appropriate management for severe cases. New strategy for early dengue infection and severity risk prediction early in the course of illness is indispensable so that management strategies can be promptly implemented.

\section{References}

Ahmed, F.U., Mahmood, C.B., Sharma, J.D., Hoque, S.M., Zaman, R. and Hasan, M.H. 2001. Dengue fever and dengue haemorrhagic fever in chidren the 2000 outbreak in Chittatong, Bangladesh. Dengue Bulletin. 25: 33-9.

Alcon, S., Talarmin, A., Debruyne, M., Falconar, A., Deubel, V. and Flamand, M. 2002. Enzyme-linked immunosorbent assay specic to dengue virus type 1 nonstructural protein NS1 reveals circulation of the antigen in the blood during the acute phase of dis-ease in patients experiencing primary or secondary infections. Journal of Clinical Microbiology. 40: 376-81.

Beltran-Silva, S.L., Chacon-Hernández, S.S., Moreno-Palacios, E. and PereyraMolina, J.A. 2016. Clinical and differential diagnosis: Dengue, chikungunya and Zika, Revista Médica del Hospital General de México.

Chadwick, D., Arch, B., Wilder-Smith, A. and Paton, N. 2006. Distinguishing dengue fever from other infections on the basis of simple clinical and laboratory features: Application of logistic regression analysis. Journal of Clinical Virology. 35: 147-53.

Chan, C.Y.Y. and Ooi, E.E. 2015. Dengue: an update on treatment options. Future
Microbiol. 10: 2017-2031.

Colombo, T.E., Vedovello, D., Pacca-Mazaro, C.C., Mondini, A., Araújo J.P., Cabrera, E., Lopes, J.C., Penha dos Santos, I.N., Negri Reis, A.F., Costa, F.R., Antônio Cruz, L.E.A., Ferreira, J.E.S., de Oliveira Rocha, E.G., Kroon, R.V., de Morais Bronzoni, N. and Vasilakis, M.L. 2016. Dengue virus surveillance: Detection of DENV-4 in the city of São José do Rio Preto, SP, Brazil, Acta Tropica. 164: 84-89.

Crance, J.M., Scaramozzino, N., Jouan, A. and Garin, D. 2003. Interferon, ribavirin, 6-azauridine and glycyrrhizin: antiviral compounds active against pathogenic flaviviruses. Antiviral Research. 58: 73-79.

Damonte, E.B., Matulewicz, M.C. and Cerezo, A.S. 2004. Sulfated seaweed polysaccharides as antiviral agents. Curr. Med. Chem. 11: 2399-2419.

Deubel, V., Laille, M., Hugnot, J.P, et al., 1990. Identication of dengue sequences by genomic amplication: rapid diagnosis of dengue virus serotypes in peripheral blood. Journal of Virology Methods. 30: 41-54.

Endy, T.P., Chunsuttiwat, S., Nisalak, A., Libraty, D. H., Green, S., Rothman, A. L., Vaughn, D. W. and Ennis, F.A. 2002. Epidemiology of inapparent and symptomatic acute dengue virus infection: a prospective study of primary school children in Kamphaeng Phet, Thailand. Ameriacn Journal of Epidemiology. 156: 40-51.

Focks, D. A. and R. Barrera. 2007. "Dengue transmission dynamics: assessment and implications for control," Scientific Working Group Report on Dengue, World Health Organization, Geneva, Switzerland.

Gurugama, P., Garg, P., Perera, J., Wijewickrama, A. and Seneviratne, S.L. 2010. Dengue viral infections. Indian 
Journal of Dermatology. 55: 68-78.

Guzman, M. G., and G. Kouri. 2003. Dengue: an update. Lancet Infectious Disease. 2: 33-42.

Henchal, E.A., Putnak , J.R. et al., 1990. dengue viruses. Clinical Microbiology Review. 1: 376-396.

Horstick, O., Runge-Ranzinger, S., M. B. Nathan, and A. 2010. Kroeger, "Dengue vector-control services: how do they work? A systematic literature review and country case studies," Transactions of the Royal Society of Tropical Medicine and Hygiene, vol. 104 (6): 379-386.

Innis, B.L., Nisalak, A. and Nimmannitya. S. 1989. An enzyme-linked immunosorbent assay to characterize dengue infections where dengue and Japanese encephalitis co-circulate. American Journal of Tropical Medicine Hygiene. 40: 418-427.

Itoda, I., Masuda, G., Suganuma, A., Imamura, A., Ajisawa, A., Yamada, $\mathrm{K}$, et al., 2006. Clinical features of 62 imported cases of dengue fever in Japan. American Journal of Tropical Medicine Hygiene. 75: 470-4.

Kuehn, B.M. 2014. Chikungunya virus transmission found in the United States: US health authorities brace for wider spread. The Journal of the American Medical Association. 27: 776-7.

Lanciotti, R.S., Calisher, C.H., Gubler, D.J., Chang, G.J. and Vorndam, A.V. 1992. Rapid detection and typing of dengue viruses from clinical samples by using reverse transcrip-tase-polymerase chain reaction. Journal of Clinical Microbiology. 30: 545-51.

McMichael, A. J., Woodru, R. E. and Hales, S. 2006. Climate change and human health: present and future risks. Lancet. 367(9513): 859-869.

Messina, J.P. Brady, O.J. and Scott T.W. et al., 2014. "Global spread of dengue virus types: mapping the 70 year history," Trends in Microbiology. 22(3): 138-146.

Morin, C.W., Comrie, A.C. and Ernst, K. 2013. Climate and dengue transmission: evidence and implications. Environ Health Perspective. 121: 1264-72.

Morin, C.W. Monaghan, A.J. Hayden, M.H. Barrera, R. and K. Ernst. 2015. Meteorologically driven simulations of dengue epidemics in San Juan, PR," PLOS Neglected Tropical Diseases. 9 (8): Article ID e0004002.

Muller, D.A., Alexandra C. I., Depelsenaire. and Young, P.Y. 2017. Clinical and Laboratory Diagnosis of Dengue Virus Infection. Dengue Virus Diagnostics. Journal of Infectious Disease.215: S89S95.

Narayanan, M., Aravind, M.A., Thilothammal, N., Prema, R., Sargunam, C.S. and Ramamurty N. Dengue fever epidemic in Chennai-a study of clinical profile and outcome. Indian Pediatr 2002;39:1027-33

Paixao, E.S., Maria Gloria Teixeira, Maria da Conceição N Costa, Laura C Rodrigues Dengue during pregnancy and adverse fetal outcomes: a systematic review and meta-analysis.

www.thelancet.com/infection Published online March 3, 2016 http://dx.doi.org/10.1016/S14733099(16)00088-8.

Radakovic-Fijan, S., Graninger, W., Müller, C., Hönigsmann, H. and Tanew, A. 2002. Dengue hemorrhagic fever in a British travel guide. Journal of American Acadamy of Dermatology. 46: 430-3.

Rezza, G. 2014. Dengue and chikungunya: long-distance spread and outbreaks in naïve areas. Path Glob Health. 108(8): 349-55.

Rigau-Perez, J.G., Clark, G.G., Gubler, D.J., Reiter, P., Sanders, E.J. and Vorndam, 
A.V. 1998. Dengue and dengue haemorrhagic fever. Lancet. 352: 97177.

Roitt, I. Brostoff, J. and Male, D. 2001. Immunology, $6^{\text {th }}$ edn. Harcourt Publishers Limited: Toronto. 240-422.

Rosenberger, K.D., Lum, L., Alexander, N., Junghanss, T., Wills, B. and Jaenisch, T. 2016. Clinical Study Group. Vascular leakage in dengue-clinical spectrum and influence of parenteral fluid therapy. Tropical Medicine \& International Health. 21: 445-53.

Roth, A., Mercier, A. and Lepers, C, et al., 2014. Concurrent outbreaks of dengue, chikungunya and zika virus infections - an unprecedented epidemic wave of mosquito-borne viruses in the Pacific 2012-2014. European Surveillance. 19: 20929.

Shivpuri, A. and Shivpuri, A. 2011. DengueAn overview. Dental Medical Problem. 48:153-6.

Shuman, E.K. 2010. Global climate change and infectious diseases. New England Journal of Medicine. 362(12): 10611063.

Shu, P.Y., Chen, L.K. and Chang, S.F, et al., 2004. Dengue virus serotyping based on envelope and membrane and nonstructural protein NS1 serotypespecic capture immunoglobulin $\mathrm{M}$ enzyme-linked immunosorbent assays. Journal of Clinical Microbiology. 42: 2489-94.

Simmons, C.P., Chau, T.N, suy T.T, et al., 2007. Maternal antibody and viral factors in the pathogenesis of dengue virus in infants. Journal of Infectious Disease. 196: 416-24.

Simmons, C.P., Farrar, J., Nguyen, V.V.C, et al., 2012. Current concepts: dengue. New England Journal of Medicine. 366: 1423-1432.

Teles, F. R. R., Prazeres, D. M. F. and Lima-
Filho, J. L. 2005. Trends in dengue diagnosis. Rev. Med. Virol. 15: 287302.

Thai, K.T.D. and Anders, K.L. 2011. "The role of climate variability and change in the transmission dynamics and geographic distribution of dengue," Experimental Biology and Medicine. 236 no. (8): 944-954.

Vasilakis, N. Cardosa, J.K.A., Hanley, E. C., Holmes. and Weaver, S. C. 2011. Fever from the forest: prospects for the continued emergence of sylvatic dengue virus and its impact on public health, Nature Reviews Microbiology. 9 (7): 532-541.

Whitehorn, J. and Farrar, J. 201. Dengue. British Medical Bulletin. 95: 161-73.

Whitehorn, J., Yacoub, S., Anders, K.L., et al., 2014. Dengue therapeutics, chemoprophy-laxis, and allied tools: state of the art and future directions. PLoS Neglected Tropical Disease. 8: 3025.

WHO, 2011. Comprehensive Guidelines for Prevention and Control of Dengue, WHO. http://apps.who.int/iris/bitstream/ 10665/204894/1/B4751.pdf.

WHO. 1997. Dengue haemorrhagic fever: diagnosis, treatment, prevention and control, 2nd ed. World Health Organization, Geneva, Switzerland.

WHO. 1999. Regional Guidelines on Dengue/DHF Prevention and Control. Regional Publication 29/1999. World Health Organization: Geneva.

WHO. 2009. Dengue: Guidelines for Diagnosis, Treatment, Prevention and Control. New ed. Geneva, Switzerland: WHO. 1-160.

WHO. 2012. Global strategy for dengue prevention and control 2012-2020. WHO. Aug:1-43.

WHO. 2015. Dengue and severe dengue. Fact Sheet No117. Updated May, 2015. http://www.who.int/mediacentre/factshe 
ets/fs117/en/ (accessed July 15, 2015).

Wilder-Smith, A., Ooi, E.E., Vasudevan, S.G. and Gubler, D.J. 2010. Update on dengue: epide-miology, virus evolution, antiviral drugs, and vaccine development. Current Infectious Disease Reports. 12: 157164

Wills BA, Dung NM, Loan HT, et al., Comparison of three fluid solutions for resuscitation in dengue shock syndrome. 2005. The New England
Journal of Medicine. 353: 877-889.

Wiwanitkit, V. 2017. Dengue fever: diagnosis and treatment. Expert Review of Antiinfective Therapy. 8:841-845.

Young, P.R., Hilditch, P.A., Bletchly, C. and Halloran, W. 2000. An antigen capture enzyme-linked immunosorbent assay reveals high levels of the dengue virus protein NS1 in the sera of infected patients. Journal of Clinical Microbiology. 38: 1053-7.

\section{How to cite this article:}

Prem Kumar, R., R. Sunith, A. Karthikeyan and Pradeep Kumar, V. 2020. Dengue Fever: A Review Article. Int.J.Curr.Microbiol.App.Sci. 9(01): 1502-1510.

doi: https://doi.org/10.20546/ijcmas.2020.901.168 